\title{
Dynamic scheduling in the engineer-to-order (ETO) assembly process by the combined immune algorithm and simulated annealing method
}

\author{
Jiang, C. ${ }^{a}{ }^{,}, \mathrm{Xi}$, J.T. ${ }^{\mathrm{a}}$ \\ ${ }^{a}$ School of Mechanical Engineering, Shanghai Jiao Tong University, Shanghai, P.R. China
}

\begin{abstract}
A B S T R A C T
With the increasing demand for customization, the engineer-to-order (ETO) production strategy plays an increasingly important role in today's manufacturing industry. The dynamic scheduling problem in ETO assembly process was investigated. We developed the mathematical model to represent the problem. In order to reduce rescheduling frequency, we introduced the concept of starting time deviation and improved the rolling horizon driven strategy. We proposed the hybrid algorithm combining immune algorithm (IA) and simulated annealing (SA) with the minimization of the rescheduling cost as the objective. The IA was designed as the global search process and the SA was introduced to improve the local searching ability. The scenario-based approach was used to model the disruptions affecting the tasks to be executed. Performance of the rolling horizon driven strategy and the hybrid algorithm were evaluated through simulations, the experiment analysis showed the best parameters of rolling horizon methods and demonstrated the feasibility of the hybrid algorithm. The hybrid algorithm was tested on different scale benchmark instances and the case that collected from a steam turbine assembly shop. The quality of solution in terms of cost obtained by the hybrid algorithm was found superior to the other three algorithms proposed in the literature.
\end{abstract}

\section{ARTICLE INFO}

Keywords:

Engineer-to-order (ETO);

Assembly process;

Dynamic scheduling;

Rescheduling;

Rolling horizon;

Immune algorithm;

Simulated annealing

*Corresponding author:

sjjzxjc@163.com

(Jiang, C.)

Article history:

Received 16 April 2019

Revised 14 September 2019

Accepted 16 September 2019

\section{References}

[1] Jana, T.K., Saha, P., Sarkar, B., Saha, J. (2013). Implementation of agent based holonic control in discrete manufacturing, Advances in Production Engineering \& Management, Vol. 8, No. 3, 157-168, doi: 10.14743/apem2013. $\underline{3.163}$.

[2] Grabenstetter, D.H., Usher, J.M. (2015). Sequencing jobs in an engineer-to-order engineering environment, Production \& Manufacturing Research, Vol. 3, No. 1, 201-217, doi: 10.1080/21693277.2015.1035461.

[3] Alfieri, A., Tolio, T., Urgo, M. (2012). A project scheduling approach to production and material requirement planning in manufacturing-to-order environments, Journal of Intelligent Manufacturing, Vol. 23, No. 3, 575-585, doi: $10.1007 / \mathrm{s} 10845-010-0396-1$.

[4] De Lit, P., Latinne, P., Rekiek, B., Delchambre, A. (2001). Assembly planning with an ordering genetic algorithm, International Journal of Production Research, Vol. 39, No. 16, 3623-3640, doi: 10.1080/00207540110056135.

[5] Alfieri, A., Tolio, T., Urgo, M. (2011). A two-stage stochastic programming project scheduling approach to production planning, The International Journal of Advanced Manufacturing Technology, Vol. 62, No. 1-4, 279-290, doi: 10.1007/s00170-011-3794-4.

[6] Hytonen, J., Niemi, E., Toivonen, V. (2008). Optimal workforce allocation for assembly lines for highly customised low-volume products, International Journal of Services Operations and Informatics, Vol. 3, No. 1, 28-39, doi: 10.1504/ijsoi.2008.017703. 
[7] Jiang, P., Ding, J.L., Guo, Y. (2018). Application and dynamic simulation of improved genetic algorithm in production workshop scheduling, International Journal of Simulation Modelling, Vol. 17, No. 1, 159-169, doi: 10.2507/IJSIMM17(1)CO3.

[8] Yang, X.P., Gao, X.L. (2018). Optimization of dynamic and multi-objective flexible job-shop scheduling based on parallel hybrid algorithm, International Journal of Simulation Modelling, Vol. 17, No. 4, 724-733, doi: 10.2507/ IJSIMM17(4)C019.

[9] Hicks, C., Song, D.P., Earl, C.F. (2007). Dynamic scheduling for complex engineer-to-order products, International Journal of Production Research, Vol. 45, No. 15, 3477-3503, doi: 10.1080/00207540600767772.

[10] Vieira, G.E., Herrmann, J.W., Lin, E. (2003). Rescheduling manufacturing systems: A framework of strategies, policies, and methods, Journal of Scheduling, Vol. 6, No. 1, 39-62, doi: 10.1023/A:1022235519958.

[11] Deblaere, F., Demeulemeester, E., Herroelen, W. (2011). Reactive scheduling in the multi-mode RCPSP, Computers \& Operations Research, Vol. 38, No. 1, 63-74, doi: 10.1016/i.cor.2010.01.001.

[12] Herroelen, W., Leus, R. (2004). Robust and reactive project scheduling: A review and classification of procedures, International Journal of Production Research, Vol. 42, No. 8, 1599-1620, doi: 10.1080/00207540310001638055.

[13] Herroelen, W., Leus, R. (2005). Project scheduling under uncertainty: Survey and research potentials, European Journal of Operational Research, Vol. 165, No. 2, 289-306, doi: 10.1016/i.ejor.2004.04.002.

[14] Demeulemeester, E., Herroelen, W., Leus, R. (2008). Proactive-reactive project scheduling, In: Artigues, C., Demassey, S., Néron, E. (eds.), Resource-constrained project scheduling: Models, algorithms, extensions and applications, Wiley-ISTE, London, United Kingdom, 203-211, doi: 10.1002/9780470611227.ch13.

[15] Van de Vonder, S., Ballestín, F., Demeulemeester, E., Herroelen, W. (2007). Heuristic procedures for reactive project scheduling, Computers \& Industrial Engineering, Vol. 52, No. 1, 11-28, doi: 10.1016/i.cie.2006.10.002.

[16] Zhu, G., Bard, J.F., Yu, G. (2005). Disruption management for resource-constrained project scheduling, Journal of the Operational Research Society, Vol. 56, No. 4, 365-381, doi: 10.1057/palgrave.jors.2601860.

[17] Chakrabortty, R.K., Sarker, R.A., Essam, D.L. (2016). Multi-mode resource constrained project scheduling under resource disruptions, Computers \& Chemical Engineering, Vol. 88, 13-29, doi: 10.1016/j.compchemeng.2016.01. $\underline{004 .}$

[18] Sonmez, R., Uysal, F. (2015). Backward-forward hybrid genetic algorithm for resource-constrained multiproject scheduling problem, Journal of Computing in Civil Engineering, Vol. 29, No. 5, Article number: 04014072, doi: 10.1061/(ASCE)CP. 1943-5487.0000382.

[19] Gholamian, M.R., Heydari, M. (2017). An inventory model with METRIC approach in location-routing-inventory problem, Advances in Production Engineering \& Management, Vol. 12, No. 2, 115-126, doi: 10.14743/apem 2017.2.244.

[20] Qin, W., Zhang, J., Song, D. (2018). An improved ant colony algorithm for dynamic hybrid flow shop scheduling with uncertain processing time, Journal of Intelligent Manufacturing, Vol. 29, No. 4, 891-904, doi: 10.1007/ s10845-015-1144-3.

[21] Deblaere, F., Demeulemeester, E., Herroelen, W. (2011). Proactive policies for the stochastic resourceconstrained project scheduling problem, European Journal of Operational Research, Vol. 214, No. 2, 308-316, doi: 10.1016/j.ejor.2011.04.019.

[22] De Castro, L.N., Von Zuben, F.J, (1999). Artificial immune systems: Part I - Basic theory and applications, Technical Report, Technical report, RT DCA 01/99, 95 pages.

[23] Metropolis, N., Rosenbluth, A.W., Rosenbluth, M.N., Teller, A.H., Teller, E. (1953). Equation of state calculations by fast computing machines, The Journal of Chemical Physics, Vol. 21, No. 6, 1087-1092, doi: 10.1063/1.1699114.

[24] Cao, Q.K., Qin, M.N., Ren, X.Y. (2018). Bi-level programming model and genetic simulated annealing algorithm for inland collection and distribution system optimization under uncertain demand, Advances in Production Engineering \& Management, Vol. 13, No. 2, 147-157, doi: 10.14743/apem2018.2.280.

[25] Jiang, C., Hu, X., Xi, J. (2019). Integrated multi-project scheduling and hierarchical workforce allocation in the ETO assembly process, Applied Sciences, Vol. 9, No. 5, 885-904, doi: 10.3390/app9050885.

[26] Wauters, T., Kinable, J., Smet, P., Vancroonenburg, W., Vanden Berghe, G., Verstichel, J. (2016). The multi-mode resource-constrained multi-project scheduling problem, Journal of Scheduling, Vol. 19, No. 3, 271-283, doi: 10.1007/s10951-014-0402-0.

[27] Mobini, M., Mobini, Z., Rabbani, M. (2011). An artificial immune algorithm for the project scheduling problem under resource constraints, Applied Soft Computing, Vol. 11, No. 2, 1975-1982, doi: 10.1016/j.asoc.2010.06.013.

[28] Józefowska, J., Mika, M., Różycki, R., Waligóra, G., Węglarz, J. (2001). Simulated annealing for multi-mode resource-constrained project scheduling, Annals of Operations Research, Vol. 102, No. 1-4, 137-155, doi: 10.1023/ A:1010954031930.

[29] Goncharov, E.N., Leonov, V.V. (2017). Genetic algorithm for the resource-constrained project scheduling problem, Automation and Remote Control, Vol. 78, No. 6, 1101-1114, doi: 10.1134/S0005117917060108. 


\section{APEM}

\title{
Dinamično načrtovanje izdelave po naročilu (ETO) v postopku montaže s kombiniranim imunskim algoritmom in simuliranim ohlajanjem
}

\author{
Jiang, C. ${ }^{\mathrm{a},{ }^{*}}, \mathbf{X i}$, J.T. $^{\mathrm{a}}$ \\ ${ }^{a}$ School of Mechanical Engineering, Shanghai Jiao Tong University, Shanghai, P.R. China
}

\section{POVZETEK}

$\mathrm{Z}$ naraščajočim povpraševanjem po prilagoditvi ima strategija izdelave po naročilu (ETO) vse pomembnejšo vlogo $v$ današnji industriji. Preučen je bil problem dinamičnega načrtovanja proizvodnje za ETO v postopku montaže. Za predstavitev problema smo razvili matematični model. Da bi zmanjšali frekvenco načrtovanja, smo uvedli koncept odstopanja po začetnem času in izboljšali strategijo kotalečega obzorja. Predlagali smo hibridni algoritem, ki združuje imunski algoritem (IA) in simulirano ohlajanje (SA) s ciljem zmanjšanja stroškov reprogramiranja. IA je bil zasnovan kot globalni iskalni postopek, SA pa je bil vključen za izboljšanje lokalne iskalne sposobnosti. Za modeliranje motenj, ki vplivajo na naloge, ki jih je treba izvesti je bil uporabljen pristop, ki temelji na scenarijih. Učinkovitost strategije kotalečega obzorja in hibridnega algoritma, smo ocenili s simulacijami, analiza eksperimenta pa je razkrila najboljše parametre metode kotalečega obzorja in potrdila izvedljivost hibridnega algoritma. Hibridni algoritem je bil preizkušen na različnih standardnih testnih primerih in na primeru montaže parnih turbin. V primerjavi s tremi algoritmi, predstavljenimi v literaturi, je kakovost rešitve glede na stroške, pridobljena s hibridnim algoritmom, boljša.
\end{abstract}

\section{PODATKI O ČLANKU}

Ključne besede:

Izdelava po naročilu (ETO);

Montaža;

Dinamično načrtovanje;

Reprogramiranje;

Kotaleče obzorje;

Imunski algoritem;

Simulirano ohlajanje

*Kontaktna oseba: sjjzxjc@163.com (Jiang, C.)

Zgodovina članka:

Prejet 16. aprila 2019

Popravljen 14. septembra 2019

Sprejet 16. septembra 2019 